\title{
Gruppenstimmung (group mood) als Erfolgsbedingung transformationaler Führung
} Ergebnisse einer empirischen Untersuchung

\author{
Sabine Boerner und Christian Freiherr von Streit
}

\begin{abstract}
Zusammenfassung. Der Beitrag untersucht den Zusammenhang zwischen transformationaler Führung, Stimmung der Gruppenmitglieder und Erfolg einer Arbeitsgruppe. Am Beispiel des Symphonieorchesters wird die Hypothese entwickelt, dass die transformationale Führung des Dirigenten nur dann positiv mit dem Erfolg des Orchesters verbunden ist, wenn zugleich die Stimmung der Orchestermusiker positiv ist. Eine empirische Untersuchung von 208 Musikern aus 22 deutschen Symphonieorchestern bestätigt den angenommenen Interaktionseffekt von transformationaler Führung durch den Dirigenten und positiver Stimmung der Musiker auf den künstlerischen Erfolg des Orchesters.

Schlüsselwörter: Gruppenstimmung, künstlerische Qualität, Orchester, transformationale Führung
\end{abstract}

Group mood as a condition for success in transformational leadership: Results of an empirical study

\begin{abstract}
The paper investigates the degree to which transformational leadership style and positive group mood positively affect the success of a work group. Analyzing the example of symphony orchestras, the authors develop the thesis that the conductor's transformational leadership style promotes the orchestra's success only if there is a high positive group mood among the orchestra musicians. An empirical study of 208 musicians from 22 German symphony orchestras confirms the assumed interaction effect of the conductor's transformational leadership style and the musicians' group mood on the performance of the orchestra.

Key words: group mood, artistic quality, orchestra, transformational leadership
\end{abstract}

Arbeitsgruppen entwickeln über Prozesse der kognitivemotionalen Selbstregulation eine spezifische Stimmung, die in der Literatur als work group mood (Bartel \& Saavedra, 2000) oder group emotion (Barsade \& Gibson, 1998) bezeichnet wird. Nachstehend wird die Frage untersucht, inwieweit eine positive Stimmung der Gruppe die Voraussetzung für einen erfolgreichen Einsatz der transformationalen Führung darstellt.

Gegenstand der Untersuchung ist die transformationale Führung eines Dirigenten im Symphonieorchester. Dieses bisher in der Führungsforschung kaum untersuchte Feld (Hunt, Stelluto \& Hooijberg, 2004) erscheint für unsere Fragestellung aus verschiedenen Gründen als fruchtbar: So lassen vorliegende Studien eine hohe Relevanz speziell transformationaler Führung im Orchester vermuten (Boerner, 2002; Boerner \& Krause, 2002; Faulkner, 1973). Darüber hinaus ist anzunehmen, dass eine positive Stimmung unter den Orchestermusikern für die künstlerische Qualität der Orchesterleistung in besonderem Maße bedeutsam ist. Zum einen ist die Mo- bilität der Orchestermitglieder in professionellen Orchestern eher gering (Boerner, 2002), so dass Symphonieorchester als Gruppen mit einer hohen zeitlichen Stabilität angesehen werden können, was die Entwicklung einer einheitlichen Gruppenstimmung begünstigt (Bartel \& Saavedra, 2000). Zum anderen ist die Aufgabeninterdependenz hier sehr hoch ausgeprägt (Boerner, 2002), was ebenfalls eine einheitliche Gruppenstimmung fördert (Bartel \& Saavedra, 2000). Schließlich ist anzunehmen, dass die Stimmung unter den Orchestermusikern zusätzlich in hohem Maße erfolgsbedeutsam ist, weil ihre spezifische Aufgabe, die Erarbeitung einer gemeinsamen Interpretation eines musikalischen Werkes, bereits als solche mit einer hohen Emotionalität verbunden ist.

Für konstruktive Kritik danken wir Herrn Prof. Dr. Diether Gebert, Frau Dipl.-Psych. Elisabeth Dütschke und zwei anonymen Gutachtern. 


\section{Transformationale Führung und Erfolg im Orchester}

In einer neueren Untersuchung des Konstrukts transformationale Führung (Bass, 1985, 1998; Burns, 1978) von Avolio, Bass und Jung (1999) erweisen sich drei Teilfacetten als empirisch relevant: Charisma, inspirierende Motivierung und intellektuelle Stimulierung.

Ein positiver Zusammenhang zwischen einer transformationalen Führung und der Leistung des Orchesters könnte darin vermutet werden, dass ein transformational führender Dirigent die Leistungsbereitschaft der Orchestermusiker in besonderer Weise stimuliert. Dies wird deutlich, wenn man sich die Besonderheit der Führungskonstellation im Orchester vergegenwärtigt: Orchestermusiker werden in der Regel nicht in die Erarbeitung einer künstlerischen Konzeption für die Interpretation eines Werkes einbezogen, sondern setzen diejenige Konzeption um, die der Dirigent ihnen vorgibt. Da professionelle Orchestermusiker hoch qualifiziert sind, stellt ein in diesem Sinne direktiver Führungsstil zunächst einen Widerspruch zu den Erkenntnissen der Führungstheorie dar und gefährdet daher tendenziell die Leistungsbereitschaft der Orchestermusiker (Boerner, 2002).

Vor diesem Hintergrund erscheint folgende Wirkung einer transformationalen Führung im Orchester denkbar: Im Gegensatz zu einem nur direktiv Führenden gibt der transformational führende Dirigent seine künstlerische Konzeption nicht einfach im Sinne eines Diktates vor, sondern vermittelt sie als eine Vision, die die geführten Orchestermusiker als intellektuelle Stimulierung und inspirierende Motivation erleben. Die intellektuelle Stimulierung besteht im Orchesterkontext insbesondere darin, bekannte Werke auf eine neuartige Weise zu interpretieren. Die Neuartigkeit bzw. Originalität der Konzeption eines Dirigenten wirkt vor allem deshalb in hohem Maße stimulierend, weil Orchestermusiker in der Regel mit einem eingeschränkten Kanon an bekannten Repertoirestücken konfrontiert sind (Dowd, Liddle, Lupo \& Borden, 2002).

Inspirierende Motivation entsteht umso mehr, je klarer und eindeutiger die künstlerische Konzeption vermittelt wird (Boerner, 2002). Auf diese Weise schafft der transformational führende Dirigent nicht nur Orientierung; durch das visionär-charismatische Moment, die intellektuelle Stimulierung und die inspirierende Motivation erhält seine künstlerische Konzeption zugleich eine hohe Überzeugungskraft und Verbindlichkeit für die Orchestermusiker. In der Empirie zeigt sich entsprechend, dass ein direktives Führungsverhalten des Dirigenten insbesondere dann die künstlerische Qualität des Orchesters fördert, wenn der Dirigent von den Musikern zugleich als unbestrittene fachliche Autorität wahrgenommen wird (Boerner \& Krause, 2002). Ebenso zeigt sich in empirischen Untersuchungen, dass die künstlerische Qualität des Orches- ters umso höher eingestuft wird, je mehr dem Dirigenten Expertenmacht und Identifikationsmacht zugeschrieben werden (Krause, Boerner, Lanwehr \& Nachtigall, 2002).

\section{Positive Gruppenstimmung und Erfolg im Orchester}

Orchester stellen vergleichsweise heterogene und große Gruppen dar. Die Musiker pflegen z. T. unterschiedliche Selbstverständnisse und fühlen sich häufig stärker ihrer Instrumentengruppe zugehörig als dem Orchester. Somit ist nicht davon auszugehen, dass sich eine gemeinsame soziale Identität der Orchestermusiker (Tajfel, 1981) gleichsam von selbst einstellt. Zudem bestehen Unterschiede in Bezug auf die orchesterinterne Hierarchiestufe und die Bezahlung zwischen Tuttisten (tutti [ital.] = alle), Stimmführern und Solisten. Weitere Unterschiede bestehen in Bezug auf Dauer der Zugehörigkeit zum Orchester, Alter, Nationalität und künstlerisches und technisches Niveau der einzelnen Musiker. Nach den Befunden der Diversity-Forschung kann eine heterogene $\mathrm{Zu}$ sammensetzung von Teams erfolgsbezogen zwar vorteilhaft sein; es können jedoch erhebliche Kooperationsbarrieren entstehen, die sich negativ auf die Gruppenleistung auswirken (Gebert, 2004; Williams \& O'Reilly, 1998).

Zugleich erfordert die simultane Interdependenz der Aufgaben (Shea \& Guzzo, 1987; Wageman, 1995) eine intensive und permanente Koordination im Orchester (Saavedra, Earley \& Van Dyne, 1993, S. 62). Während bei einer sequenziellen Interdependenz der Aufgaben lediglich die Teilergebnisse der einzelnen Mitwirkenden koordiniert werden müssen, sind bei simultaner Interdependenz unmittelbar die von den verschiedenen Musikern arbeitsteilig ausgeführten Teilprozesse zu koordinieren (Saavedra, Earley \& Van Dyne, 1993, S. 62). Voraussetzung für eine hohe künstlerische Leistung des Orchesters ist daher ein exakt synchronisiertes Spiel (Boerner, 2004): Ein einheitliches Klangbild des Orchesters etwa lässt sich nur dann erreichen, wenn alle beteiligten Musiker z.B. bei einer Steigerung der Lautstärke (crescendo) jeweils ihre individuelle Lautstärke zur selben Zeit und im selben Maße steigern.

Inhaltlich lässt sich die Koordination des Musizierens dabei nicht auf eher technisch erscheinende Aspekte wie den gemeinsamen Beginn oder das gemeinsame Ende des Spiels reduzieren. Von wesentlicher Bedeutung für die Orchesterleistung ist die Koordination im künstlerischen Sinne: Eine gemeinsame Steigerung der Lautstärke oder eine gemeinsame Artikulation setzen vor allem einen emotionalen Gleichklang der Musiker voraus. Erst dieser emotionale Gleichklang ermöglicht einen musikalischen Gleichklang des Orchesters, der für eine stimmige Interpretation notwendig ist (Boerner, 2004). 
An dieser Stelle wird die Bedeutung der positiven Stimmung unter den Musikern erkennbar. Speziell eine positiv gefärbte Gruppenstimmung erweist sich in der Literatur generell als förderlich für die wechselseitige Hilfsbereitschaft der Gruppenmitglieder, etwa im Sinne des Organizational Citizenship Behavior (Müller \& Bierhoff, 2001). Der auf der Individualebene berichtete Befund, dass positive Affekte mit prosozialem Verhalten verbunden sind (Isen \& Baron, 1991), wird damit auch auf der Ebene von Arbeitsgruppen bestätigt.

Speziell im Orchester ist anzunehmen, dass der emotionale Gleichklang der Musiker dazu beiträgt, dass sie zu einem Klangkörper verschmelzen und in der Lage sind, wie ein einziges Instrument zu reagieren. Emotionaler Gleichklang und wechselseitiges Verständnis erleichtern insbesondere die nonverbale Kommunikation der Musiker untereinander (Williamon \& Davidson, 2002). Dies ist die Voraussetzung dafür, dass ein virtuos spielender, transformational führender Dirigent seine künstlerischen Vorstellungen ohne Abstriche realisieren kann. Daraus lässt sich ableiten:

Hypothese: Der Zusammenhang zwischen transformationaler Führung und der künstlerischen Qualität des Orchesters wird durch die Gruppenstimmung wie folgt moderiert: Je stärker die transformationale Führung durch den Dirigenten bei gleichzeitig positiver Stimmung unter den Musikern, desto besser die künstlerische Qualität des Orchesters.

\section{Methode}

\section{Stichprobe}

Zur Untersuchung der obigen Hypothese wurden in den Jahren 2002 und 2003208 Musiker (70\% männlich, $30 \%$ weiblich) aus 22 deutschen Symphonieorchestern (durchschnittliche Mitgliederzahl: 98 Musiker) befragt (Rücklaufquote $76 \%$; von Streit, 2003). Es wurde keine reine Zufallsstichprobe gezogen; angesichts der begrenzten Grundgesamtheit wurde versucht, die Teilnahmebereitschaft durch die persönliche Ansprache der Vorstände von 29 Berufsorchestern zu erhöhen, die den Fragebogen an maximal 12 Musiker ihres Orchesters weiterleiteten. Das Durchschnittsalter der Befragten liegt bei 40 Jahren $(S D=8.83)$ mit einer durchschnittlichen Erfahrung als Berufsmusiker im Orchester von 15 Jahren $(S D=9.39)$. Die Zusammensetzung nach Instrumentengruppen (62\% Streicher, 36\% Bläser, 2\% Schlagwerk) und Position (67\% Tuttisten, 33\% Solisten bzw. Stimmführer) entspricht in etwa der tatsächlichen Verteilung in einem Orchester.

\section{Instrumente}

Für die Befragung wurde ein Instrument entwickelt, das die transformationale Führung durch den Dirigenten, die Stimmung der Musiker sowie die künstlerische Qualität des Orchesters misst. Die Verwendung selbst formulierter, kontextspezifischer Skalen erschien vielversprechender für die Erklärung der abhängigen Variablen als die Verwendung generalisierter Instrumente, weil in der Empirie dasselbe Führungsverhalten in Bezug auf unterschiedliche Zielkriterien und in unterschiedlichen situativen Kontexten unterschiedliche Wirkungen entfaltet (Gebert, 2002). Da die Stimmung der Musiker und die künstlerische Qualität einer Aufführung nur auf der Ebene des Orchesters sinnvoll zu bestimmen sind, wurden die Daten hier nicht auf der Ebene des Individuums verrechnet; die Antworten der Musiker wurden pro Orchester zu je einem Mittelwert für die auf einer 7-stufigen Likert-Skala gemessenen Konstrukte (transformationale Führung, Stimmung der Musiker, künstlerische Qualität des Orchesters) aggregiert. Um die Zulässigkeit der Aggregation der individuellen Antworten auf Orchesterniveau zu überprüfen, wurde der Koeffizient $r_{\mathrm{WG}(J)}$ verwendet (James, Demaree \& Wolf, 1984). Dieses Verfahren erlaubt abhängig von der Übereinstimmung der Antworten der Befragten pro Orchester die Aggregation der Daten (Bliese, 2000; Kozlowski \& Hattrup, 1992, S. 162).

Die transformationale Führung wurde in enger Anlehnung an die Konzeption von Avolio et al. (1999) operationalisiert. Da die drei Skalen durchschnittlich mit etwa $r=.80$ korrelieren (vgl. Avolio et al., 1999), wurde hier von einem eindimensionalen Konstrukt ausgegangen, und es wurden zwei Items für jede der drei Facetten Charisma (,... hat Charisma“; „Wir sind stolz, mit ihm zusammenzuarbeiten"), inspirierende Motivation $(, \ldots$ verdeutlicht seine Ziele mit Bildern und Gesten“; „,macht uns klar verständlich, was er von uns will“") und intellektuelle Stimulierung (,....macht oft völlig neue, überzeugende Interpretationsvorschläge“; „ermöglicht uns, bekannte Werke in einem neuen Licht zu sehen“) formuliert. Die Stimmung der Orchestermusiker wurde über acht Items erhoben (,zerstritten“ [R], „sympathisch“, „unfähig“ $[R]$, ,halten gut zusammen“, ,,angenehm“, „,faul“ [R], ,es gibt hier Spannungen zwischen den Instrumentengruppen“ $[R]$, „, die verschiedenen Gruppen im Orchester halten gut zusammen"). ${ }^{1}$

Als Maß für die Leistung wurde hier die künstlerische Qualität des Orchesters verwendet. In Anlehnung an frühere Studien (Boerner \& Krause, 2002; Krause, Boerner, Lanwehr \& Nachtigall, 2002) wurde hier nicht die künstlerische Qualität selbst zu messen versucht, sondern primär die Reaktion Dritter auf die Leistung des Orchesters: „Das Publikum beurteilt die Qualität unseres

\footnotetext{
${ }^{1}[\mathrm{R}]=$ Die Items wurden in umgepolter Form verrechnet.
} 
Tabelle 1. Deskriptive Statistiken und Interkorrelationen aller Untersuchungsvariablen

\begin{tabular}{llllll}
\hline Variable & $M$ & $S D$ & 1 & 2 & 3 \\
\hline 1. künstlerische Qualität & 5.58 & 0.59 & $(.73)$ & & \\
2. transformationale Führung & 4.57 & 1.45 & $.38^{\#}$ & $(.94)$ & \\
3. positive Gruppenstimmung & 4.64 & 0.84 & $.56^{* *}$ & $.42^{\#}$ & $(.94)$ \\
$r_{\mathrm{WG}(\mathrm{J})}{ }^{\mathrm{a}}$ & & & .92 & .82 & .88 \\
\hline
\end{tabular}

Anmerkungen. $N=22$; in der Hauptdiagonalen sind in Klammern die internen Konsistenzen (Cronbachs Alpha) dargestellt; ${ }^{\mathrm{a}} r_{(\mathrm{WG})}=\mathrm{Koeffizient}$, der die Zulässigkeit der Aggregierung der Daten auf Orchesterniveau prüft; ${ }^{*} p<.10,{ }^{* *} p<.01$.

Tabelle 2. Multiple Regressionsanalysen zur Vorhersage der künstlerischen Qualität im Orchester

\begin{tabular}{lll}
\hline Schritt und Variable & Modell 1 & Modell 2 \\
\hline positive Gruppenstimmung & $.48^{* *}$ & $.48^{* *}$ \\
transformationale Führung & .18 & .14 \\
positive Gruppenstimmung $\times$ & & $.51^{* *}$ \\
transformationale Führung & & \\
$R^{2}$ & $.34^{*}$ & $.60^{* *}$ \\
$\Delta R^{2}$ & & $.26^{* *}$ \\
\hline
\end{tabular}

Anmerkungen. $N=22$ bei allen Analysen; dargestellt sind standardisierte Beta-Gewichte; $* p<.05, * * p<.01$.

Orchesters in der Regel als...“; „Andere Mitwirkende (z.B. Solisten) beurteilen die Qualität unseres Orchesters in der Regel als...".

Zusätzlich wurde nach der Qualität des Orchesters im Vergleich zu anderen Orchestern derselben Tarifklasse gefragt. Diese Einschätzung wurde pro Orchester von mindestens zwei weiteren Musikern vorgenommen, die nicht bereits das Führungsverhalten und die Gruppenstimmung eingestuft hatten.

\section{Ergebnisse}

Tabelle 1 zeigt deskriptive Statistiken und Interkorrelationen aller Untersuchungsvariablen.

Die Hypothese wurde in einer multiplen hierarchischen Regression überprüft (vgl. Tabelle 2). Dabei wurden im ersten Modell nur die Prädiktoren aufgenommen; im zweiten Modell wurde zur Analyse der angenommenen Wechselwirkung zusätzlich das Produkt aus transformationaler Führung und positiver Gruppenstimmung aufgenommen, wobei die Variablen zentriert wurden (Aiken \& West, 1991).

In beiden Modellen wird der Haupteffekt transformationaler Führung erwartungsgemäß nicht signifikant. Hypothesenkonform zeigt sich, dass aus der Aufnahme

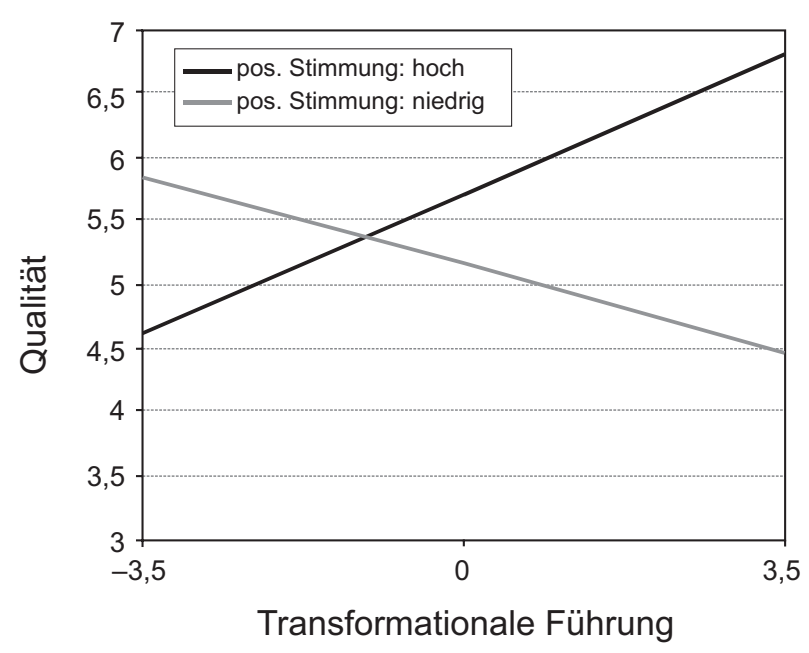

Abbildung 1. Wechselwirkung von transformationaler Führung des Dirigenten und positiver Gruppenstimmung unter den Musikern auf die künstlerische Qualität des Orchesters.

Anmerkungen. Separate Regressionslinien für hohe bzw. niedrige positive Stimmung der Gruppe (eine Standardabweichung über respektive unter dem Skalenmittelwert).

des Interaktionsterms aus transformationaler Führung und positiver Gruppenstimmung ein signifikanter Anstieg, $F(1,18)=11.75, p<.001$, des erklärten Varianzanteils auf $R^{2}=.60$ resultiert. Die Form der signifikanten Interaktion wird in Abbildung 1 veranschaulicht. Zusätzlich wird in beiden Modellen der Haupteffekt der positiven Gruppenstimmung der Musiker auf die künstlerische Qualität des Orchesters signifikant $(\beta=.48, p<.01)$.

\section{Diskussion}

Die vorliegenden Befunde bestätigen die zentrale Hypothese der Untersuchung: Ein positiver Zusammenhang zwischen der transformationalen Führung des Dirigenten und der künstlerischen Qualität des Orchesters besteht nur dann, wenn zugleich eine positive Stimmung unter 
den Orchestermusikern herrscht. Unter dieser Voraussetzung lässt sich über ein hohes $\mathrm{Ma} ß$ an transformationaler Führung des Dirigenten eine hohe künstlerische Qualität des Orchesters vorhersagen.

Die Relevanz einer positiven Stimmung der Orchestermusiker für den Erfolg des Orchesters wird durch einen zusätzlichen Befund unterstrichen: Obwohl die $\beta$ Koeffzienten bei Vorliegen einer signifikanten Interaktion nicht als Haupteffekte interpretiert werden können (Jaccard \& Turrisi, 2003, S. 24), mag der signifikante Haupteffekt der Gruppenstimmung in beiden Modellen ein gelegentlich berichtetes Phänomen widerspiegeln: In einem sehr gut eingespielten Team - das heißt, bei positiver Gruppenstimmung - sind Musiker mitunter auch unabhängig vom Dirigenten zu einer hohen künstlerischen Leistung im Stande und können daher z.B. einen weniger guten Dirigenten weitgehend ignorieren.

Im Sinne einer theoretischen Schlussfolgerung aus der Studie erscheint die Tatsache bedeutsam, dass die erfolgsförderliche Wirkung transformationaler Führung nicht voraussetzungslos, sondern an die Bedingung einer positiven Stimmung der Geführten gebunden ist. Angesichts der Spezifika der untersuchten Stichprobe stellt sich die Frage nach der Generalisierbarkeit der hier vorgestellten Befunde auf organisationale Arbeitsgruppen. Im Sinne des kontingenztheoretischen Ansatzes ist daher systematisch zu überlegen, welche Randbedingungen für die Gruppe Orchester kennzeichnend sind. Dabei erscheint es unrealistisch, die hier geltende spezifische Konstellation aus situativen Randbedingungen als Ganze auf betriebliche Kontexte zu übertragen; vielmehr erscheint eine gesonderte Betrachtung der einzelnen Randbedingungen zielführend (Boerner, 2002).

Die überdurchschnittliche Gruppengröße und -heterogenität legen eine Übertragung auf die Zusammenarbeit zwischen betrieblichen Gruppen bzw. auf Organisationen mit flachen Hierarchien nahe (Hunt, Stelluto \& Hooijberg, 2004). Aufgrund der hohen Professionalität der Geführten und der Kreativität bzw. Emotionalität der Aufgaben erwarten Hunt et al. (2004, S. 157 f.) von der Erforschung des Führungsverhaltens im Orchester darüber hinaus Aussagen zur Führung in New-wave-Organisationen wie F\&E-Abteilungen, professionellen Dienstleistungen und akademischen Institutionen. Die außergewöhnlich hohe Aufgabeninterdependenz im Orchester lässt dagegen nur in Grenzen Übertragungen auf andere inhaltliche Felder mit durchgehend hohem Koordinationsbedarf (wie z.B. im Katastrophenschutz) zu; vielmehr erscheint hier eine fallweise Differenzierung angebracht: So sind in professionellen Teams (wie z. B. in Krankenhäusern, Forschungsteams etc.) Situationen denkbar, in denen temporär eine hohe Aufgabeninterdependenz vorliegt.

Abschließend ist in methodischer Hinsicht einschränkend anzumerken, dass ein Längsschnittdesign, das ab- hängige und unabhängige Variablen zeitlich versetzt erhebt, aussagekräftiger gewesen wäre als das hier verwendete Querschnittsdesign. Da die hier untersuchte Stichprobe von $N=22$ Orchestern relativ klein ist und daher Schwankungen der Parameterschätzungen unterworfen ist, wäre der gefundene Moderatoreffekt in einer größeren Stichprobe zu überprüfen. Problematisch bleibt zusätzlich die Messung der künstlerischen Qualität des Orchesters. Aufbauend auf den Erfahrungen früherer Studien (Boerner \& Krause, 2002; Krause, Boerner, Lanwehr \& Nachtigall, 2002) wurde in dieser Untersuchung durch die Einbeziehung zusätzlicher unabhängiger Einstufungen der künstlerischen Qualität das Problem des single subject method consistency bias (McDonald, 1999) zwar nicht vollständig umgangen, aber zumindest entschärft. Die hier verwendete Kombination der Datenquellen zur künstlerischen Qualität gefährdet allerdings tendenziell die Reliabilität des Konstrukts. Wünschenswert wäre in künftigen Untersuchungen daher eine Erhebung der künstlerischen Qualität ausschließlich aus der Perspektive zusätzlicher Informationsquellen.

\section{Literatur}

Aiken, L. S. \& West, S. G. (1991). Multiple regression: Testing and interpreting interactions. Newbury Park, CA: Sage.

Avolio, B. J., Bass, B. M. \& Jung, D. I. (1999). Re-examining the components of transformational and transactional leadership using the Multifactor Leadership Questionnaire. Journal of Occupational and Organizational Psychology, $72,441-462$

Barsade, S. G. \& Gibson, D. E. (1998). Group emotion: A view from top and bottom. In M. A. Neale \& E. A. Mannix (Eds.), Research on managing groups and teams (pp.81102). Stanford, CT: JAI Press.

Bartel, C. A. \& Saavedra, R. (2000). The collective construction of work group moods. Administrative Science Quarterly, 45, 197-231.

Bass, B. M. (1985). Leadership and performance beyond expectations. New York: Free press.

Bass, B. M. (1998). Transformational leadership: Industrial, military, and educational impact. Rahwah, NY: Erlbaum.

Bliese, P. (2000). Within-group agreement, non-independence, and reliability. In K. J. Klein \& S. W. J. Kozlowski (Eds.), Multilevel theory, research, and methods in organizations (pp.349-381). San Francisco: Jossey-Bass.

Boerner, S. (2002). Führungsverhalten und Führungserfolg ein Beitrag zu einer Theorie der Führung am Beispiel des Musiktheaters. Wiesbaden: Gabler.

Boerner, S. (2004). Artistic quality in an opera company - towards the development of a concept. Nonprofit Management and Leadership, 4, 425-436.

Boerner, S. \& Krause, D. E. (2002). Führung im Orchester: Kunst ohne künstlerische Freiheit? Eine empirische Untersuchung. Zeitschrift für Personalforschung, 16, 90-106.

Burns, J. M. G. (1978). Leadership. New York: Harper \& Row.

Dowd, T., Liddle, K., Lupo, K. \& Borden, A. (2002). Organizing the musical canon: The repertoires of major U.S. symphony orchestras, 1842 to 1969 . Poetics, 30, 35-61. 
Faulkner, R. R. (1973). Orchestra in interaction: Some features of communication and authority in an artistic organization. The Sociology Quarterly, 14, 147-157.

Gebert, D. (2002). Führung und Innovation. Stuttgart: Kohlhammer.

Gebert, D. (2004). Innovation durch Teamarbeit - eine kritische Bestandsaufnahme. Stuttgart: Kohlhammer.

Hunt, J. G., Stelluto, G. E. \& Hooijberg, R. (2004). Toward new-wave organization creativity: Beyond romance and analogy in the relationship between orchestra-conductor leadership and musician creativity. Leadership Quarterly, $15,154-162$.

Isen, A. M. \& Baron, R. A. (1991). Positive affect as a factor in organizational behavior. In B. M. Staw \& L. L. Cummings (Eds.), Research in organizational behavior (Vol. 13; pp. 1-53). Greenwich, CT: JAI Press.

Jaccard, J. \& Turrisi, R. (2003). Interaction effects in multiple regression (2nd ed.). Thousand Oaks: Sage University Press.

James, L. R., Demaree R. G. \& Wolf, G. (1984). Estimating within-group interrater reliability with and without response bias. Journal of Applied Psychology, 69, 85-98.

Kozlowski, S. W. \& Hattrup, K. (1992). A disagreement about within-group agreement: Disentangling issues of consistency versus consensus. Journal of Applied Psychology, $77,161-167$.

Krause, D. E., Boerner, S., Lanwehr, R. \& Nachtigall, C. (2002). Strukturgleichungsmodelle zur Analyse von Macht und Führungserfolg im Orchester. Zeitschrift für Personalpsychologie, 1, 124-137.

McDonald, R. P. (1999). Test theory: A unified treatment. Mahwah, NJ: Lawrence Erlbaum.

Müller, G. F. \& Bierhoff, H. W. (2001). Stimmungseinflüsse in Projektgruppen. In R. Fisch, D. Beck \& B. Englich (Hrsg.), Projektgruppen in Organisationen (S. 323-336). Göttingen: Verlag für Angewandte Psychologie.
Saavedra, R., Earley, P. C. \& Van Dyne, L. (1993). Complex interdependence in task-performing groups. Journal of Applied Behavioral Psychology, 78, 61-72.

Shea, G. P. \& Guzzo, R. A. (1987). Groups as human resources. In K. M. Rowland \& G. P. Ferris (Eds.), Research in personnel and human resources management (pp.323-356). Greenwich, CT: JAI Press.

Streit, C. von (2003). Untersuchung zum Arbeitsklima in deutschen Kulturorchestern - eine bundesweite Fragebogenstudie. Unveröffentlichte Diplomarbeit, Freie Universität Berlin.

Tajfel, H. (1981). Human groups and social categories: Studies in Social Psychology. Cambridge: Cambridge University Press.

Wageman, R. (1995). Interdependence and group effectiveness. Administrative Science Quarterly, 40, 145-180.

Williamon, A. \& Davidson, J. W. (2002). Exploring co-performer communication. Musicae Scientiae, 1, 53-72.

Williams, K. Y. \& O’Reilly, C. A. (1998). Demography and diversity in organizations. Research in Organizational Behavior, 20, 77-140.

Eingegangen: 12.01.2004

Revision eingegangen: 01.06.2004

Prof. Dr. Sabine Boerner

Universität Konstanz, Fachbereich Politik- und Verwaltungswissenschaft, Lehrstuhl für Management, insbesondere Strategie und Führung Universitätsstraße 10

78457 Konstanz

E-Mail: sabine.boerner@uni-konstanz.de 